\title{
OVERVIEW OF REWRITABLE OPTICAL TECHNOLOGY AND MARKET (Invited)
}

\author{
Y. AOKI \\ MO Business Development Division, Storage Systems Group, Sony Corporation, Atsugi, Japan
}

In the 1970's, many research groups in the world were investigating magneto-optical media for rewritable storage applications[1-4]. In 1980, KDD Research Laboratory developed the world's first successful device for recording, readout, and erasure of a magneto-optical (MO) disk using a laser diode[5]. The objective was to combine the higher recording density of optical disks with the rewritability of magnetic media.

In 1983, Sony and KDD announced that they had succeeded in developing a magneto-optical disk using TbFeCo that can be recorded on and erased with a laser diode[6-8]. KDD evaluated this system for mass storage of computing data in its international telecommunication services.

Since 1985, many companies released technology announcements for a magneto-optical disk; however, MO disks did not come out as real products for commercial use because of the lack of standards. For storage devices with removable media, standards which allow interchangeability are extremely important to drive the acceptance of a product in the marketplace.

During this period, ANSI took leadership in standardization of 5.25- inch optical disk interchangeability, followed by JNC, ECMA, and ISO. The standardization of 5.25-inch magneto-optical disk was especially accelerated since the computer industry had been anxiously awaiting the commercial availability of rewritable optical disk products.

The controversy in the choice of servo format unfortunately led to ISO's acceptance of both continuous-composite (CCS) format and sampled servo (SS) format. However, because ANSI only accepted the CCS format, most drive manufacturers were motivated to introduce CCS-based MO drives. So far ten drive manufacturers have introduced CCS format drives and only one manufacturer has introduced SS format drives in the market. 
Magneto-optical disk systems are finding great acceptance in the computer storage marketplace. The major advantages of $\mathrm{MO}$ systems are large data capacity and the disk interchangeability, which allows users to distribute software. Major applications are CAD/CAM/CAE, database management, electronic publishing, LAN services, and scientific and medical imaging.

By the end of 1990 , it is estimated that over 100,000 units of 5.25 inch MO drives will be installed in the market. Fifty percent of those will be in the U.S., thirty-five percent in Japan, and fifteen percent in Europe.

In 1991, 3.5-inch magneto-optical disk systems will be introduced by several companies. For the 3.5 inch-MO disk, ISO has a single format (CCS format) and the data capacity is 128 MBytes. Its market size is expected to be about 50,000 units for the first year and over 200,000 units for the second year.

Although the MO systems have many advantages, in some cases the data rate is slower and the access time is longer than that for hard disk drive. In this talk, an overview of magneto-optical disk business and several recent technical innovations to overcome the demerits of the current MO systems mentioned above are discussed, such as direct overwriting and higher density recording technologies.

\section{References}

[1] P. Chaudhari, J. J. Cuomo, and R. J. Gambino, Appl. Phys. Lett., 22, 337 (1973).

[2] D. Chen, Applied Optics, 13, 767 (1974).

[3] N. Imamura, Y. Mimura, and T. Kobayashi, Jpn. J. Appl. Phys., $\underline{15}, 179$ (1976).

[4] Y. Togami, Appl. Phys. Lett., 10, 323 (1978).

[5] N. Imamura and C. Ota, Jpn. J. Appl. Phys., 19, L731 (1980).

[6] F. Tanaka, Y. Nagao, and N. Imamura, IEEE Trans. Mag., MAG20, 1022 (1984).

[7] S. Miyaoka, S. Sato, and Y. Aoki, CLEO Tech. Digest, Tu-P4 (1984).

[8] Y. Aoki, T. Ihashi, N. Sato, and S. Miyaoka, IEEE Trans. Mag., MAG-21, 1624 (1985). 